\title{
Study on Restraining Cavitation of Axial Piston Pump Based on Structure of Cylinder Block and Valve Plate Triangular Throttling Groove
}

\author{
Sun Zegang $\mathbb{D}^{1,2}$ Li Yundong, ${ }^{1,2}$ Liang Ning, ${ }^{1,2}$ and Zhong Hongmin ${ }^{1,2}$ \\ ${ }^{1}$ School of Mechanical Engineering, Sichuan University of Science and Engineering, Zigong 643000, China \\ ${ }^{2}$ Sichuan Provincial Key Lab of Process Equipment and Control, Zigong 643000, China \\ Correspondence should be addressed to Sun Zegang; szg527@126.com
}

Received 9 September 2021; Revised 3 December 2021; Accepted 6 December 2021; Published 25 January 2022

Academic Editor: Mahdi Mohammadpour

Copyright (c) 2022 Sun Zegang et al. This is an open access article distributed under the Creative Commons Attribution License, which permits unrestricted use, distribution, and reproduction in any medium, provided the original work is properly cited.

Raising the rotational speed is an effective way to improve the power density of axial piston pump, but high rotational speed tends to cause plunger cavity and valve plate throttling groove cavitation. The cavitation of axial piston pump must reduce its volumetric efficiency and cause vibration and noise. In order to reduce the cavitation of axial piston pump and improve the performance of axial piston pump, the optimization of anticavitation structure parameters of cylinder block and valve plate throttling groove is studied in this paper. Firstly, according to the condition of no cavitation in the piston cavity fluid, the mathematical model of the ratio of plunger cavity diameter to axial piston pump cylinder block kidney hole hydraulic diameter is established. It is found that when the ratio is small, the cavitation degree of the piston cavity is weakened. Secondly, this paper analyzes and studies the influence of the valve plate triangular throttling groove structure parameters on the cavitation flow of the triangular throttling groove. It is found that reducing the structural parameters $\gamma$ or $\theta$ can reduce the cavitation intensity of the triangular throttling groove fluid. Finally, combined with the analysis result of the ratio of piston cavity between the piston cavity diameter and the cylinder block kidney hole hydraulic diameter and the research results of the anticavitation of the valve plate triangular throttling groove, the optimized structure of anticavitation of the axial piston pump is proposed, and the simulation results show that the improved axial piston pump has better anticavitation performance.

\section{Introduction}

An axial piston pump is the power source and the heart of the hydraulic system. The main performance indexes of an axial piston pump are outlet flow stability, cavitation, vibration, noise, volumetric efficiency, and so on. Cavitation is one of the main factors that affect the function of an axial piston pump. The plunger cavity and triangular throttling groove cavitation must result in a reduction of an axial piston pump volumetric efficiency and an increase of its vibration. The cavitation at the interface between the cylinder block and valve plate that could cause the valve plate wear was studied by Zecchi and Ivantysynova [1]. The axial piston pump cavitation that could cause the flow pulsation increase was studied by Zhang [2]. Therefore, it is critical to study the plunger cavity and triangular throttling groove cavitation mechanism and to explore the method of decreasing or eliminating the axial piston pump cavitation.

Many investigations have focused on axial piston pump cavitation. With the rapid development of computer simulation technology, the computational fluid dynamics (CFD) method has been widely introduced to the investigation of hydrofoil and pump cavitation by many scholars in recent years, such as Liu et al. [3], Liu et al. [4, 5], Sun and Tan [6], and Berta et al. [7]. Kassem and Bahr [8] established the mathematical model describing the piston cavity pressure variations to study an axial piston pump cavitation. Nie and Li [9] thought that the valve plate geometry and its overcurrent area could greatly affect the plunger cavity cavitation and thought that increasing the inlet pressure of an axial 
piston pump could greatly reduce the plunger cavity cavitation degree. Bergada et al. [10] concentrated on the study of the influence of the plunger cavity and valve plate structure on the axial piston pump cavitation. Liu et al. [11] studied the cavitation erosion mechanism of an axial piston pump and thought that the cavitation erosion depended not only on the values of pressure and speed of the pressure damping groove but also on the direction of velocity. Liu et al. [12] presented the method that the impingement angle should be restricted in the range of $30^{\circ} \sim 60^{\circ}$ during the early stage of oil backflushing, according to the research results of axial piston pump cavitation erosion mechanism. Liu et al. [13] studied the damping hole of the valve plate and thought that the damping hole near the outlet pressure damping groove has little effect on the jetting angle of the pressure damping groove and that the structure of the outlet pressure damping groove has to be changed to reduce the cavitation erosion on the high-pressure pump port plate through changing the jetting angle of the outlet pressure damping groove. Vacca et al. [14] illustrated a numerical methodology for the description of the effective flow rate of an axial piston pump and studied the effect of the cavitation of an axial piston pump on its effective flow. Jian et al. [15] simulated and analyzed a water axial piston pump plunger cavity cavitation by FLUENT. They found that increasing the inlet pressure of the water axial piston pump could reduce the water axial piston pump cavitation. Liu et al. [16] believed that an axial piston pump cavitation was affected by many factors, such as the rotation rate of the cylinder block, inlet pressure, and the valve plate geometry. Richardson et al. [17] put forward that the cavitation at interface between the cylinder block and the valve plate could affect the motion of the floating valve plate. Ivantysynova et al. [18] presented a new design method for valve plate design and provided a direction for anticavitation design of axial piston pump. Kuma et al. [19] and Zhang et al. [20] studied the plunger cavity cavitation and put forward a method that the use of a valve plate groove tends to prevent plunger cavity cavitation from appearing. Zhou et al. [21] presented a new approach to model cavitation due to air release and oil evaporation in lumped parameter modeling schemes, and they take as a particular reference the case of external gear pumps. Changbin et al. [22] and Casoli et al. [23] studied the cavitation of axial piston pump through the change of outlet flow or pressure ripple.

In recent years, the optimization of the anticavitation structure of axial pump has attracted many scholars' attention. Hong and Wang [24] optimized the geometry of an axial piston pump short oil absorbing hole using a genetic algorithm and found that the optimized structure was beneficial to restrain the plunger cavity cavitation. Wang [25] established the mathematical model between the plunger cavity pressure and cylinder barrel rotation angle and got the optimized geometry structure of the valve plate which could increase the anticavitation performance of an axial piston pump. In order to reduce the outlet pressure pulsation and the axial piston pump cavitation, Mandal et al. [26] put forward a methodology for designing a swashplate axial piston pump whose barrel kidneys were wider than the bridges separating the kidney ports on the plate and the pump has the ability to restrain cavitation. Mandal et al. [27] optimized the structure of the in-line axial piston pump with a pressure compensator to reduce its cavitation. Kollek et al. [28] focused on the study of the cavitation diagnosis using the audible diagnostic signal. Marinaro et al. [29] proposed a lumped parameter numerical model to optimize the axial piston pump.

It is interesting to note that the previous investigators primarily focused on an axial piston pump cavitation mechanism and restraining cavitation methods. To the best knowledge of the author, few people investigated the restraining cavitation of axial piston pumps using structural optimization methods. The goal of the paper is to study the valve plate and cylinder block geometry effect on anticavitation in an axial piston pump using theoretical and finite volume simulation methods. This paper takes a certain type of axial piston pump as a research object. Its structure is shown in Figure 1. The inclination angle of the swash plate is $15^{\circ}$. The cross angle of $\alpha$ is $4^{\circ}$. The number of the pistons is 9; that is, $i=9$. The piston diameter is $21 \mathrm{~mm}$. The nine-piston distribution circle diameter is $81 \mathrm{~mm}$. The content of this paper mainly included several aspects: (1) The ratio equation of the cylinder block kidney shape hole hydraulic diameter to the plunger cavity diameter is established for no plunger cavity cavitation. (2) The influence of the valve plate triangular throttling groove geometric parameters on an axial piston pump cavitation is studied, and the axial piston pump anticavitation structural optimization scheme is put forward.

\section{Axial Piston Pump Fluid Cavitation}

In this paper, the mixture model of FLUENT software is used to simulate the axial piston pump cavitation flow, and the gas volume fraction of the fluid is used to quantitatively judge the degree of cavitation of the axial piston pump fluid. The large gas volume fraction of fluid means a high cavitation degree of fluid.

\subsection{Axial Piston Pump Fluid Cavitation Simulation Model.} The flow channel geometry and its mesh model of an axial piston pump are established by Gambit software, as shown in Figures 2 and 3. In Figure 3, nine plunger cavities of the cylinder block are rotated around the $z$-axis, and the movement speed of the piston is expressed as

$V_{Z i}=R \omega \tan \beta \sin \left(\frac{\omega t+i \pi}{9}\right)-R \omega \tan \alpha \cdot \cos \left(\frac{\omega t+i \pi}{9}\right)$.

The axial piston pump inlet and outlet are set as pressure inlet and pressure outlet, respectively. The inlet pressure and the outlet pressure are determined as $1.01 \mathrm{E} 5 \mathrm{~Pa}$ and $3 \mathrm{MPa}$. The value of the gas volume fraction of axial piston pump inlet and outlet fluid is zero. The compressibility of the liquid and gaseous fluid and the piston velocity is set through UDF (user-defined function) by using the commercial FLUENT software, and its mixture model, RNG k- $\varepsilon$ model, dynamic mesh, and sliding mesh are used in the axial piston pump 


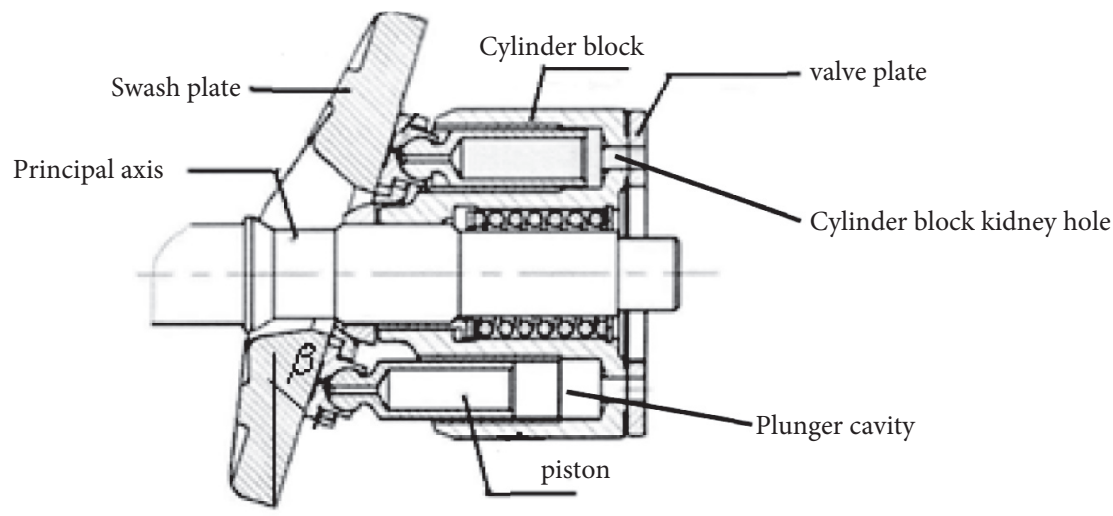

FIgURE 1: Structural representation of an axial piston pump [30].

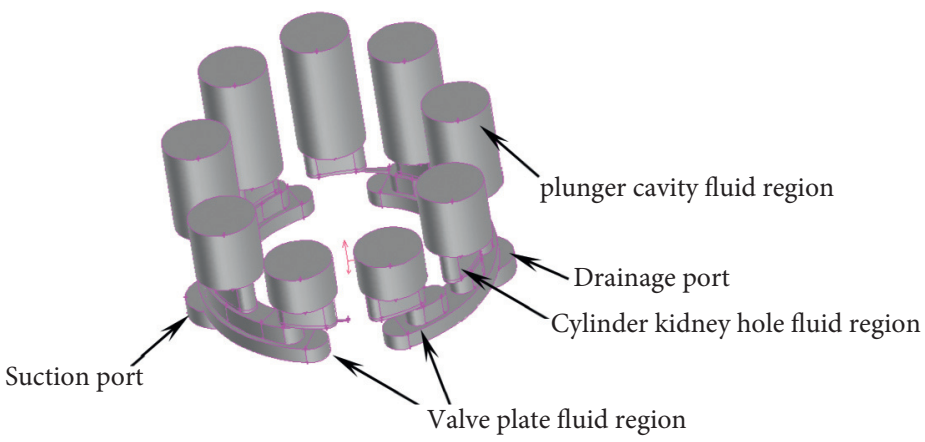

FIGURE 2: Internal fluid in an axial piston pump [30].
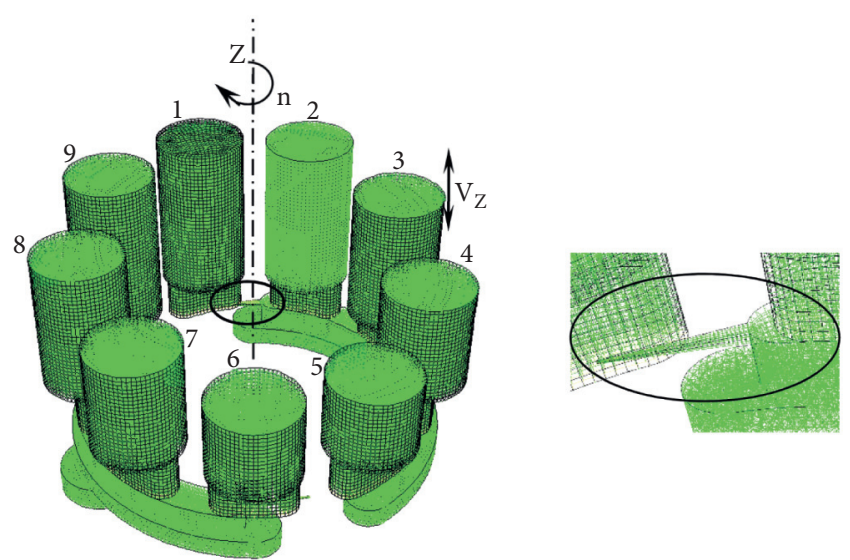

FIGURE 3: Internal flow channel mesh division of axial piston pump [30].

finite element simulation model. The dynamic mesh can be used to simulate the piston motion, and the sliding mesh can be used to simulate the relative rotation of the cylinder block and the valve plate. The control equations are space dispersed by volume method and nonstaggered grid. The twoorder upwind scheme is adopted in the turbulent kinetic energy scheme. The SIMPLE algorithm is used in the velocity component in the momentum equation and pressure coupling problem. Every iterative calculation accuracy is set as 10-8, and the time step is set as 10-4. The hydraulic oil air apart pressure is $4000 \mathrm{~Pa}$, and its saturation vapor pressure is
$2400 \mathrm{~Pa}$. The hydraulic oil density is $870 \mathrm{Kg} / \mathrm{m} 3$, and its dynamic viscosity is $0.026 \mathrm{~Pa} \cdot \mathrm{S}$. The rotation speed of the cylinder block is $1500 \mathrm{r} / \mathrm{min}$.

The calculated results of $\alpha_{v}$ and the corresponding mesh are listed in Table 1 . The values of $\alpha_{v}$ remain constant when the mesh number reaches $7.4 \mathrm{X} 105$. According to the mesh independence test, the mesh number of 155300 is finally selected in consideration of numerical accuracy and calculation cost.

2.2. Cavitation of the Plunger Cavity. Figures 4 and 5 show that the plunger cavity of an axial piston pump shows the cavitation when the plunger cavity sucks fluid. Due to the high rotation speed of the cylinder block, the fluid in the plunger cavity changes its pressure distribution under the action of centrifugal force. The fluid pressure is high on the outer side of the plunger cavity and low on the inner side. Therefore, the cavitation of the plunger cavity is mainly concentrated in the fluid inside it.

\section{Research on Suppression of Fluid Cavitation in Plunger Cavity by Cylinder Block Kidney Shape Hole Geometrical Structure}

3.1. Mechanism of the Plunger Cavity Fluid Cavitation. When the plunger cavity sucks fluid, the piston moves rapidly, the volume of the plunger cavity increases rapidly, 
TABLe 1: Axial piston pump mesh independence test.

\begin{tabular}{lcr}
\hline Item & Elements & $\alpha_{v}$ \\
\hline Mesh 1 & 46363 & 0.173 \\
Mesh 2 & 74533 & 0.162 \\
Mesh 3 & 155300 & 0.162 \\
Mesh 4 & 204735 & 0.161 \\
\hline
\end{tabular}
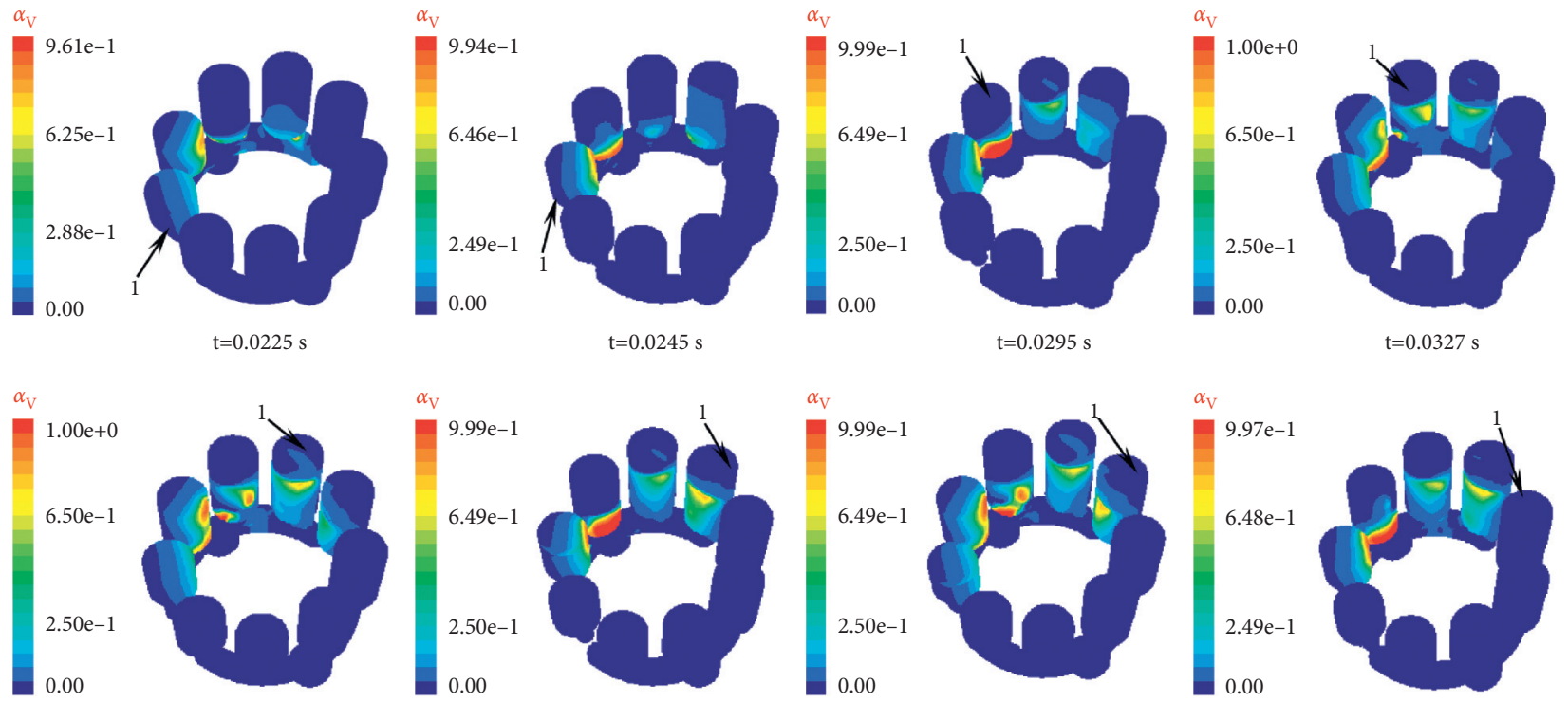

$\mathrm{t}=0.041 \mathrm{~s}$

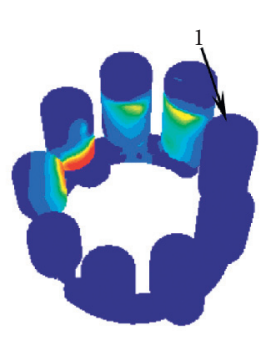

FIgURE 4: Distribution contours of gas volume fraction of no.1 plunger cavity [30].

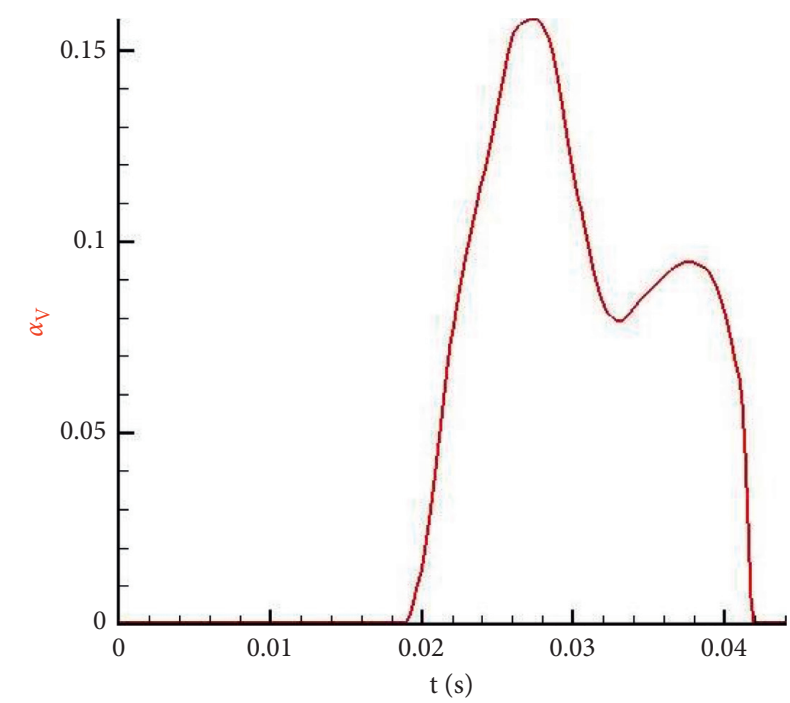

Figure 5: Curve chart of the mean value of volume fraction of no. 1 plunger cavity [30].

and the plunger cavity pressure decreases rapidly. The fluid flows into the plunger cavity through the cylinder block kidney shape hole, as shown in Figure 6. In Figure $6, D_{1}$ is the hydraulic diameter of the cylinder block kidney shape hole, $D_{2}$ is the diameter of the plunger cavity, and $\mathrm{d} l$ is the piston displacement. The volume of the fluid flowing into the

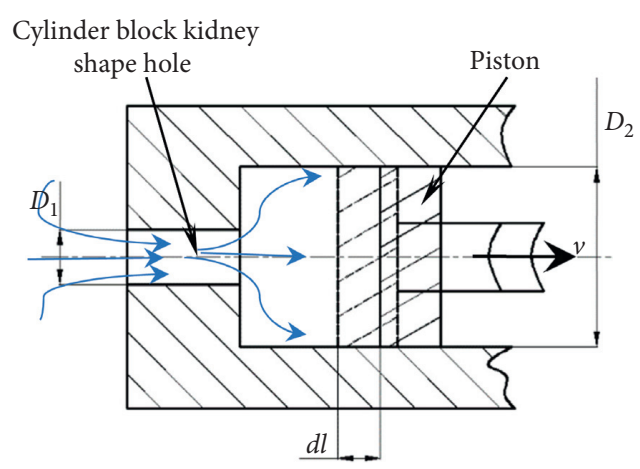

FIgURE 6: Plunger cavity suction fluid schematic diagram.

plunger cavity cannot fully fill the volume swept by the plunger. At this time, the plunger cavity fluid pressure decreases sharply, and the cavitation occurs.

3.2. Mathematical Model of the Ratio of $D_{1}$ to $D_{2}$. Taking the plunger cavity fluid as the object of analysis, its mechanical model is shown in Figure 7. In Figure 7, $P \lambda$ is the plunger cavity fluid inlet pressure, and $P$ is the pressure acting on the fluid by piston.

The piston moving velocity, piston displacement, and piston acceleration along $z$-axis are shown in equations (1)-(3). 


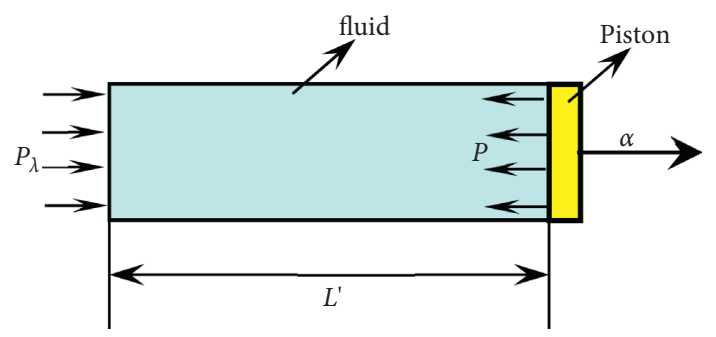

FIGURE 7: Plunger cavity fluid mechanical model.

$$
\begin{aligned}
& S_{Z}=R \tan \beta(1-\cos (\omega t))-R \tan \alpha \sin (\omega t), \\
& a_{Z}=R \omega^{2} \tan \beta \cos (\omega t)+R \omega^{2} \tan \alpha \sin (\omega t),
\end{aligned}
$$

where $\beta$ is the obliquity of the swashplate, $\alpha$ is the stagger angle of the swashplate, and $\omega$ is the angular velocity of the cylinder block.

The fluid flow through the cylinder block kidney hole in $\mathrm{d} t$ seconds can be derived as

$$
q^{\prime}=C_{d} A \sqrt{\frac{2 \cdot \Delta p}{\rho}} \mathrm{d} t .
$$

In equation (4), $A$ is the overflow area of the cylinder block kidney shape hole and can be derived as

$A=\pi / 4 D_{1}^{2}$, where $D_{1}$ is the cylinder block kidney hole hydraulic diameter.

The fluid that flows into the plunger cavity in $\mathrm{d} t$ seconds can be derived as

$$
q=\frac{\pi}{4} D_{2}^{2} V_{Z} \mathrm{~d} t
$$

In equation (5), $D_{2}$ is the plunger cavity diameter and $V_{Z}$ is the plunger velocity.

To make the plunger cavity have no void or no fluid cavitation, $q$ and $q^{\prime}$ must be equaled, so $\triangle p$ is expressed as

$$
\Delta p=\frac{m^{4} V_{Z}^{2} \rho}{2 C_{d}^{2}} .
$$

In equation (6), $m=D_{2} / D_{1}$.

The $m$ value is derived from equation (6):

$$
m=\sqrt[4]{\frac{2 \cdot \Delta p \cdot C_{d}^{2}}{V_{Z}^{2} \cdot \rho} .}
$$

As shown in Figure 7, the force balance equation of the plunger cavity fluid is

$$
p_{\lambda} \cdot S=p \cdot S+\rho \cdot L l \cdot S \cdot a .
$$

In equation (8), $p_{\lambda}$ is the fluid inlet pressure of the plunger cavity, $L^{\prime}$ is the length of the fluid in the plunger cavity, $\alpha$ is the acceleration of the plunger, and $S$ is the plunger cavity cross-sectional area.

Then, the expression of $\triangle p$ can be obtained as follows:

$$
\Delta p=p_{\lambda}-p=\rho \cdot L \prime \cdot a .
$$

In equation (9), $L \prime=S_{Z}$ and $a=a_{Z}$.

Then, the calculation formula of $\triangle p$ is as follows:

$$
\begin{aligned}
\Delta p= & \rho \cdot R(\tan \beta(1-\cos (\omega t))-\tan \alpha \cdot \sin (\omega t)) \\
& \cdot R \omega^{2}(\tan \beta \cos (\omega t)+\tan \alpha \cdot \sin (\omega t)) .
\end{aligned}
$$

When there is no cavitation empty in the plunger cavity, the critical value of $m$ is $m_{c} . m_{c}$ is calculated as follows:

$$
\begin{gathered}
m_{c}=\sqrt[4]{\frac{2 \cdot(\tan \beta(1-\cos (\omega t))-\tan \alpha \sin (\omega t))}{(\tan \beta \sin (\omega t)-\tan \alpha \cos (\omega t))^{2}}} \\
\cdot \sqrt[4]{(\tan \beta \cos (\omega t)+\tan \alpha \sin (\omega t)) C_{d}^{2}} .
\end{gathered}
$$

When $C_{d}=C_{q c}=0.85, \alpha=4^{\circ}$, and $\beta=15^{\circ}$, the curve of $m_{c}$ changes with time is shown in Figure 8.

Figures 4 and 8 show that the fluid cavitation of the plunger cavity mainly occurs between $0.013 \mathrm{~s}$ and $0.033 \mathrm{~s}$. Figure 8 shows that the $m_{c}$ value varies with time, but $m$ is constant in the practical application for an axial piston pump, so it is very difficult for an axial piston pump to meet no plunger cavity fluid cavitation in the whole operation cycle. From Figure 8 , when the $m$ value is very small, there is no fluid cavitation of the plunger cavity. So, it is impractical to make the plunger cavity fluid cavitation disappear completely.

\section{Study on Valve Plate Triangular Throttling Groove Fluid Cavitation Suppression}

4.1. Valve Plate Triangular Throttling Groove Fluid Cavitation Mechanism. When the plunger cavity crosses over the top dead center of the valve plate, the fluid of the drainage side of the pump instantly flows back into the plunger cavity. Similarly, when the plunger cavity crosses over the bottom dead center, the fluid of the plunger cavity is poured into the suction side of the pump instantaneously. In both cases, the pressure between the two ends of the valve plate triangular throttling groove is very large, and the fluid will flow through the triangular throttling groove at high speed. At this point, the kinetic energy of the fluid increases, its potential energy decreases, and the fluid pressure decreases. When the fluid pressure is lower than its air apart pressure or saturation vapor pressure, the gas nuclei in the fluid will expand to form bubbles or the gas dissolved in the fluid will be released to form bubbles, and finally, the triangular throttling groove cavitation appears.

4.2. The Valve Plate Triangular Throttling Groove Fluid Cavitation Simulation and Analysis. Figure 9 shows the valve plate triangular throttling groove, the kidney hole, and cylinder block kidney hole fluid channel geometric model and their mesh. The calculation equation of the simulation model is the equation of continuous motion of fluid and Reynolds equation. The RNG $\mathrm{k}-\varepsilon$ model and the SIMPLE algorithm are adopted. The inlet pressure is set to $20 \mathrm{MPa}$, and the outlet pressure is a standard atmospheric pressure. The hydraulic oil density is $870 \mathrm{Kg} / \mathrm{m} 3$, and its dynamic 


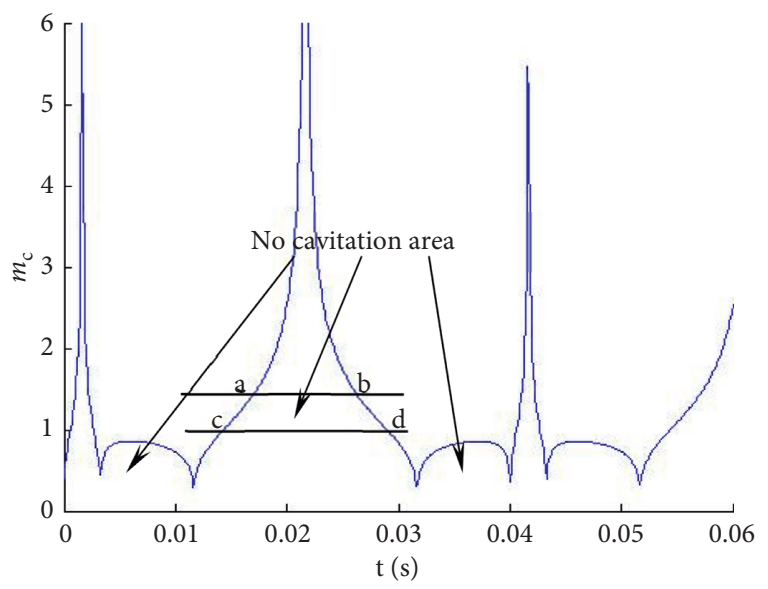

Figure 8: The change curve of the value of $m_{c}$ with time.
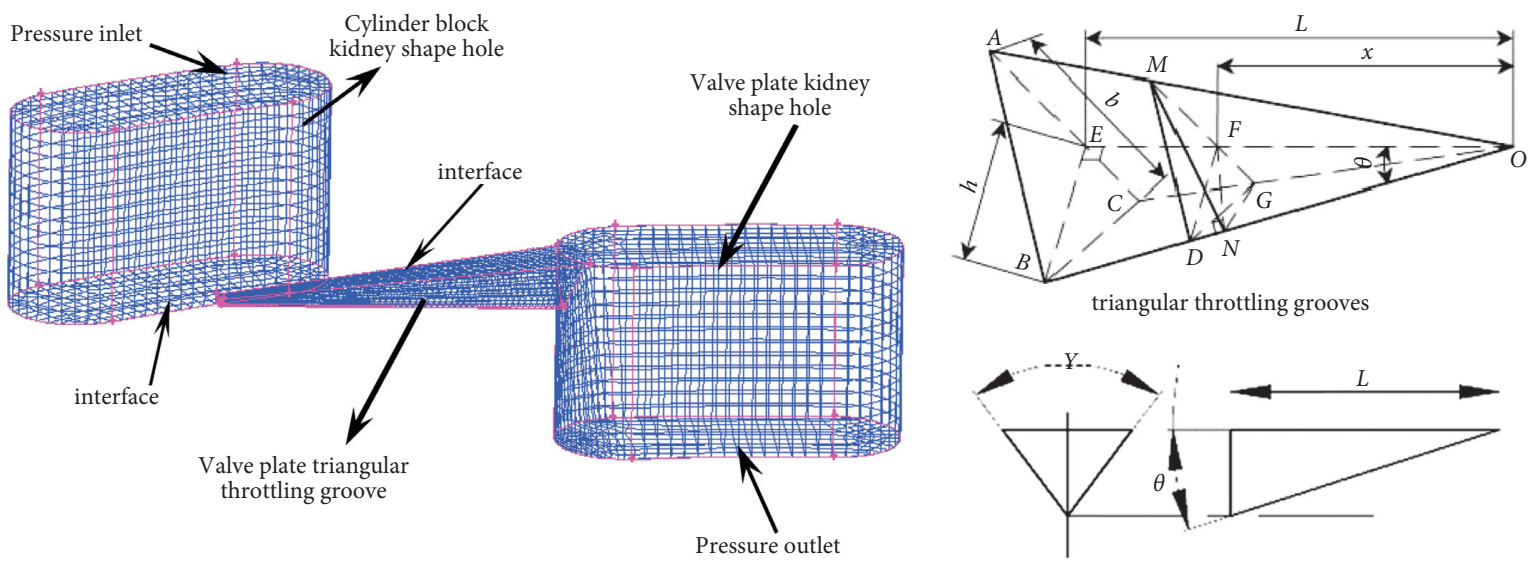

FIgURE 9: Valve plate triangular throttling groove cavitation simulation model.

viscosity is $0.026 \mathrm{~Pa} \cdot \mathrm{S}$. The hydraulic oil air apart pressure is $4000 \mathrm{~Pa}$, and its saturation vapor pressure is $2400 \mathrm{~Pa}$. The curves of $\alpha_{v}$ of the fluid in the valve plate groove and cylinder barrel kidney shape hole change with the iteration number can be monitored by using the monitoring function of the FLUENT software.

According to the mesh independence test in Table 2, the mesh number of 40315 is finally selected in consideration of numerical accuracy and calculation cost.

Figures 10 and 11 show curves of $\alpha_{v}$ of valve plate triangular throttling groove and its kidney shape hole fluid with the change of $\gamma$ or $\theta$.

From Figures 10 and 11, with the increase of the value of $\gamma$ or $\theta, \alpha_{v}$ of valve plate triangular throttling groove and its kidney shape hole fluid has been increased in varying degrees, but the increase range is different. Clearly, the change of $\theta$ has a greater effect on $\alpha_{v}$ than the change of $\gamma$. The depth of the triangular throttling groove of the valve plate is small. When $\theta$ is small, the pressure recovery of the groove fluid is faster, and the bubbles which are released from the fluid are fewer. But the small change of $\theta$ must result in the depth of the valve plate triangular throttling groove increasing in a larger gradient, and the recovery velocity of the fluid pressure is slowed down. Thus, the fluid cavitation degree of
TABLE 2: Valve plate triangular throttling mesh independence test $\left(\gamma=50^{\circ}\right.$ and $\left.\theta=4^{\circ}\right)$.

\begin{tabular}{lcc}
\hline Item & Elements & $\alpha_{v}$ \\
\hline Mesh 1 & 10430 & 0.0186 \\
Mesh 2 & 27534 & 0.0175 \\
Mesh 3 & 40315 & 0.0175 \\
Mesh 4 & 49875 & 0.0170 \\
\hline
\end{tabular}

the triangular throttling groove increases with the increase of $\theta$. Figure 10 shows that the increase of $\gamma$ has little effect on the fluid cavitation of the triangular throttling groove. This is mainly because the increase of the $\gamma$ value must lead to the increase of the lateral space of the triangular throttling groove to a certain extent, but the increase of volume is not significant, which does not affect the triangular throttling groove fluid pressure recovery speed.

4.3. Valve Plate Triangular Throttling Groove Restraining Fluid Cavitation Scheme. The results of Section 4.2 show that the larger the value of $\theta$, the larger the mean value of the gas volume fraction of the valve plate triangular throttling groove fluid. Therefore, in order to reduce the cavitation of 


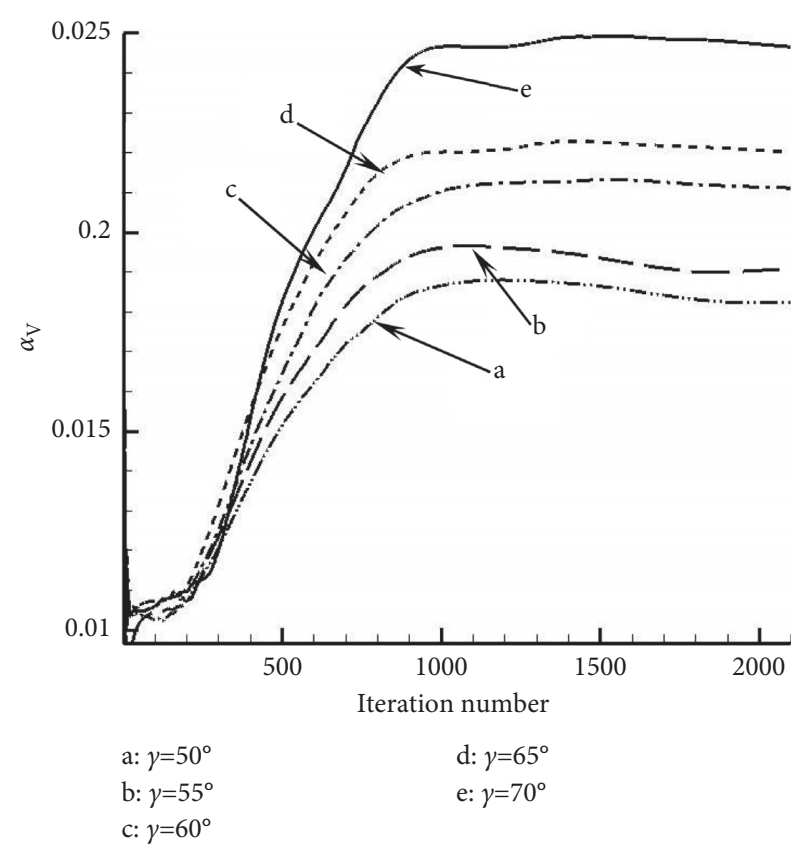

FIGURE 10: Curves of valve plate triangular throttling groove and its kidney shape hole $\alpha_{v}$ with the change of $\gamma$.

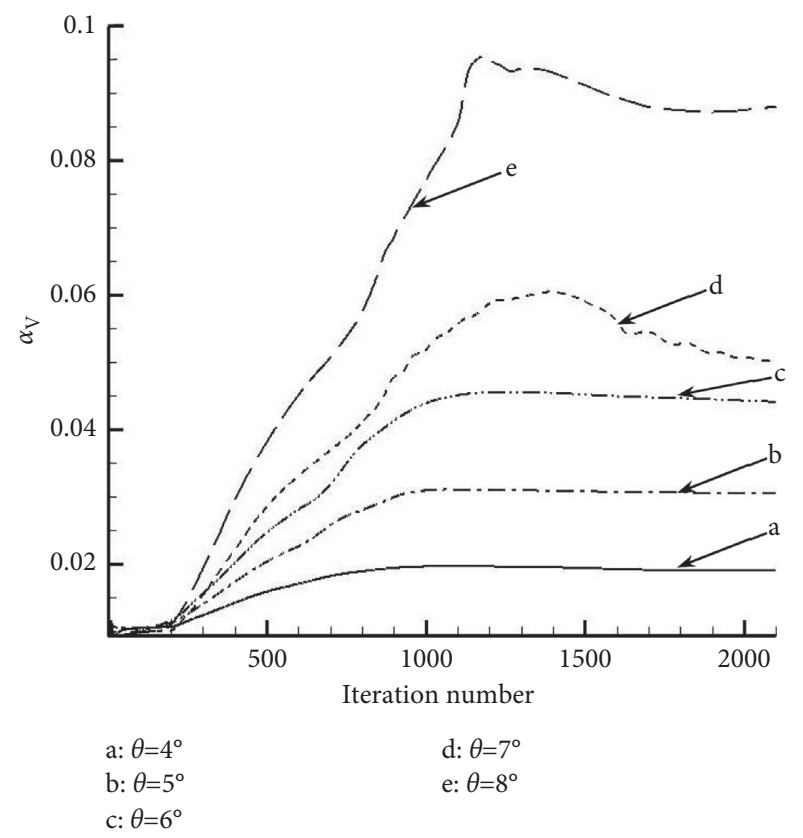

FIGURE 11: Curves of valve plate triangular throttling groove and its kidney shape hole $\alpha_{v}$ with the change of $\theta$.

the valve plate triangular groove, the scheme that the $m$ value should be as small as possible is proposed. However, the overflow area of the triangular groove remains unchanged. The object of structure improvement is the valve plate triangular throttling groove, and its main structure parameters are $\gamma=60^{\circ}$ and $\theta=6^{\circ}$.

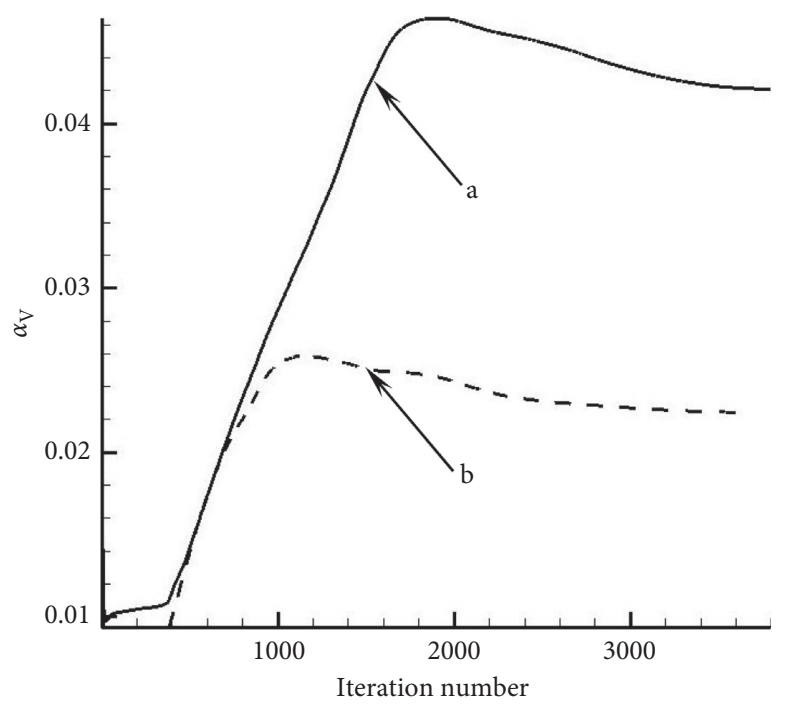

a $\gamma=60^{\circ}, \theta=6^{\circ}$

b $\gamma=104.8^{\circ}, \theta=4^{\circ}$

Figure 12: $\alpha_{v}$ change diagram of the two kinds of triangular throttling grooves and their kidney shape hole fluid when piston crosses over the bottom dead center.

The formula of triangular throttling groove overflow area is as follows:

$$
A_{V}=\frac{b x^{2} \sin (\arctan h / L)}{2 L} .
$$

In equation (12), $b$ is triangular throttling groove width, $h$ is triangular throttling groove height, $x$ is triangular throttling groove opening, and $L$ is triangular throttling groove length.

For the main structure parameters of the triangular throttling groove, according to the scheme of restraining fluid cavitation of the triangular throttling groove, $\theta$ of the triangular throttling groove is changed from $6^{\circ}$ to $4^{\circ}$. At the same time, to keep the triangular throttling groove overflow area unchanged, the structure parameter $\gamma$ is calculated as $104.8^{\circ}$ according to formula (12).

4.4. Verify the Optimization Scheme of the Valve Plate Triangular Throttling Groove Restraining Cavitation. The simulation object is two triangular throttling grooves. Their main structure parameters, respectively, are $\gamma=60^{\circ}$ and $\theta=6^{\circ} ; \gamma=104.8^{\circ}$ and $\theta=4^{\circ}$. The simulation results are shown in Figures 12 and 13. Figure 12 shows that $\alpha_{v}$ of the triangular throttling groove fluid with $\gamma=104.8^{\circ}$ and $\theta=4^{\circ}$ is nearly 0.02 lower than that of the triangular throttling groove fluid with $\gamma=60^{\circ}$ and $\theta=6^{\circ}$. Therefore, the triangular throttling groove with a smaller $\theta$ value has good restraining cavitation performance.

Figure 13 shows that the triangular throttling groove with $\gamma=104.8^{\circ}$ and $\theta=4^{\circ}$ has better restraining cavitation performance than that with $\gamma=60^{\circ}$ and $\theta=6^{\circ}$ when the piston crosses over the top dead center, but the effect is not obvious. 


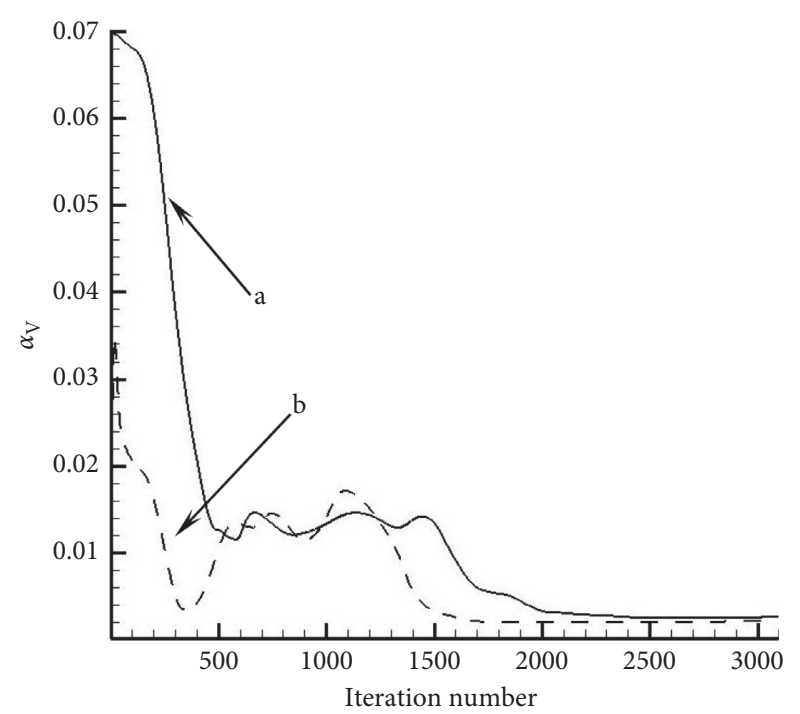

a $\gamma=60^{\circ}, \theta=6^{\circ}$

b $\gamma=104.8^{\circ}, \theta=4^{\circ}$

FIGURE 13: $\alpha_{v}$ change diagram of two kinds of triangular throttling grooves and their kidney shape hole fluid when piton crosses over the top dead center.

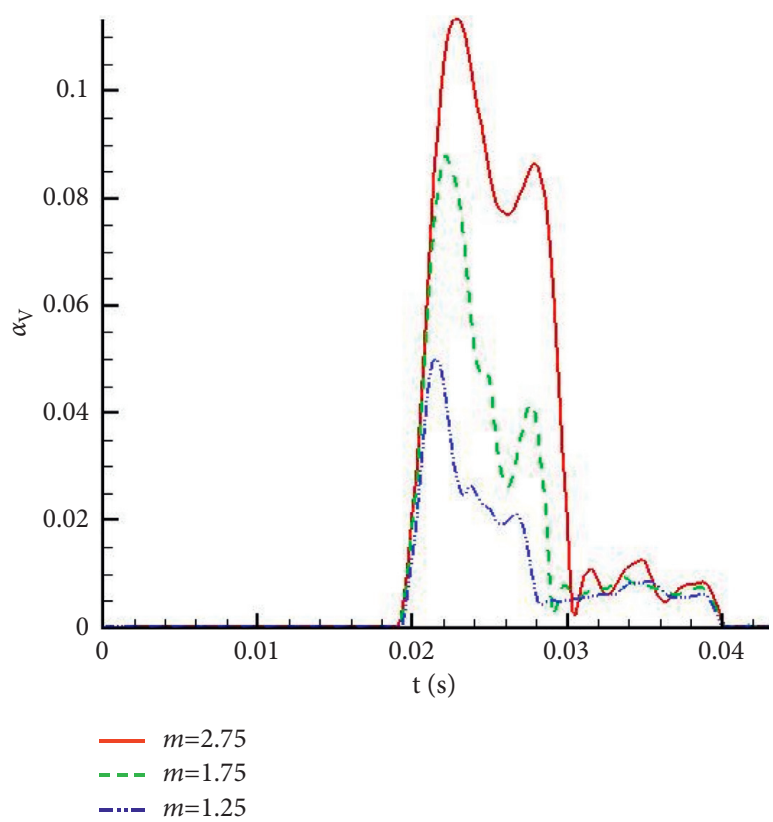

FIGURE 14: Change curve of $\alpha_{v}$ of plunger cavity at different value of $m$.

\section{Simulation Verification of the Effect of the Value of $\boldsymbol{m}$ on Plunger Cavity Cavitation}

The simulation object is the axial piston pump with triangular throttling groove structure parameters $\gamma=104.8^{\circ}$ and $\theta=4^{\circ}$, and the values of $m$ are $1.25,1.75$, and 2.75 , respectively. The inlet pressure is atmospheric pressure, the outlet pressure is $10 \mathrm{MPa}$, and the rotation speed of the cylinder barrel is $1500 \mathrm{r} / \mathrm{min}$. The change of the mean value of the gas volume fraction of the plunger cavity fluid is set as the monitoring object, and the simulation results are shown in Figure 14. From Figure 14, the larger the value of $m$ is, the larger $\alpha_{v}$ of the plunger cavity fluid is.

\section{Conclusion}

In this paper, the influence of the triangular throttling groove structural parameters on pump cavitation is analyzed by FLUENT software. The triangular throttling groove fluid cavitation degree decreases with the decrease of $\gamma$ and $\theta$, and the change of $\theta$ has the most significant effect on the triangular throttling groove fluid cavitation degree. An optimization scheme of anticavitation structure is proposed to reduce the value of $\theta$ as much as possible while keeping the triangular throttling groove overflow area unchanged. According to the optimization scheme of the triangular throttling groove of the valve plate, the triangular throttling groove with $\gamma=60^{\circ}$ and $\theta=6^{\circ}$ is optimized to the triangular throttling groove with $\gamma=104.8^{\circ}$ and $\theta=4^{\circ}$.

In this paper, a mathematical model of the $m_{c}$ under the ideal working condition of no plunger cavity fluid cavitation is established and it is found that the value of $m_{c}$ is not constant. In order to reduce the cavitation of the plunger cavity, the value of $m$ shall be taken as small as possible. Since the $m$ value cannot be too small, it is impossible to eliminate the plunger cavity fluid cavitation.

The research results of this paper provide a theory and method for the optimization of the anticavitation structure of axial piston pump. Combined with the research results of this paper, it is found that the axial piston pump with $\gamma=104.8^{\circ}, \theta=4^{\circ}$, and $m=1.25$ has the optimal anticavitation characteristics.

\section{Nomenclature}

$\alpha_{v}: \quad$ Average value of gas volume fraction

$\gamma$ : Included angle of the valve plate triangular throttling groove

$L: \quad$ Total opening of triangular throttling groove

$\theta$ : Inclination angle of triangular throttling groove

$R: \quad$ Distribution radius of the plunger cavity

$\omega$ : Angular velocity of the cylinder block

$\beta$ : Inclination angle of the swashplate

$i$ : $\quad$ Number of the plunger cavities

$D_{1}$ : Hydraulic diameter of cylinder kidney hole

$D_{2}$ : Diameter of plunger cavity

$\mathrm{d} l$ : $\quad$ Piston moving distance

$p \lambda$ : $\quad$ Fluid inlet pressure of plunger cavity

$P$ : $\quad$ Fluid pressure acting on plunger

$L^{\prime}$ : The length of the plunger cavity fluid

a) Acceleration of the piston

$a_{z}$ :

$S_{z}$ : $\quad$ Displacement of plunger

$V_{z}$ : Velocity of the piston

$q$ : The flow that flows into the plunger cavity during $\mathrm{d} t$ seconds

$q^{\prime}$ : The flow that passes through the cylinder block kidney shape hole during $\mathrm{d} t$ seconds 
A: The overflow area of cylinder block kidney shape hole

$\rho$ : The fluid density

$C_{d}$ : The outflow coefficient

$C_{q c}$ : The critical state coefficient without cavitation

$\triangle p$ : The pressure difference between two ends of cylinder block kidney shape hole

$m$ : The radio of the diameter of the plunger cavity to the hydraulic diameter of the cylinder block kidney shape hole

$m_{c}$ : The radio of the diameter of the plunger cavity to the hydraulic diameter of the cylinder block kidney shape hole without plunger cavity cavitation

$S$ : The section area of the piston

$p_{d a}$ : Absolute pressure

$p_{v}$ : $\quad$ Fluid saturated vapor pressure.

\section{Data Availability}

The data used to support the findings of this study are included within the article.

\section{Conflicts of Interest}

The authors declare that they have no conflicts of interest.

\section{Acknowledgments}

This work was financially supported by the National Natural Science Foundation of China (no. 11872043), the Talent Introduction Project of Sichuan University of Science and Engineering (no. 2018RCL11), and the project of the Sichuan Provincial Key Lab of Process Equipment and Control (no. GK201801), and the project of Sichuan Provincial Department of Science and Technology (no. 2021ZHCG0037).

\section{References}

[1] M. Zecchi and M. Ivantysynova, "A novel approach to predict the cylinder block/valve plate interface performance in swash plate type axial piston machines," ASME/Bath Symposium on Fluid Power Motion and Control, pp. 13-28, 2012.

[2] H. Zhang, "Cavitation effect to the hydraulic piston pump flow pulsation," Applied Mechanics and Materials, vol. 599601, pp. 230-236, 2014.

[3] W. T. Su, X. B. Li, and F. C. Li, "Large eddy simulation of pressure fluctuations at off-design condition in a Francis turbine based on cavitation model," Materials Science and Engineering, vol. 52, pp. 1-8, Article ID 022032, 2013.

[4] M. Liu, L. Tan, and S. Cao, "Dynamic mode decomposition of cavitating flow around ALE 15 hydrofoil," Renewable Energy, vol. 139, pp. 1-21, 2019.

[5] M. Liu, L. Tan, and S. Cao, "Cavitation-vortex-turbulence interaction and one-dimensional model prediction of pressure for hydrofoil ALE 15 by large eddy simulation," Journal of Fluids Engineering, vol. 141, no. 3, pp. 1-17, Article ID 021103, 2019.

[6] W. Sun and L. Tan, "Cavitation-vortex-pressure fluctuation interaction in a centrifugal pump using bubble rotation modified cavitation model under partial load," Journal of
Fluids Engineering, vol. 142, no. 5, pp. 1-11, Article ID 051206, 2020.

[7] G. L. Berta, P. Casoli, and A. Vacca, "Simulation model of axial piston pumps inclusive of cavitation. ASME international mechanical engineering congress \& exposition," New Orleans Louisiana, pp. 1-9, 2002.

[8] S. A. Kassem and M. K. Bahr, "Effect of port plate silencing grooves on performance of swash plate Axial piston pumps," in Proceedings of the Current Advances in Mechanical Design and Production VII. on Current Advances in Mechanical Design and Production Seventh Cairo University International MDP Conference, pp. 139-148, Cairo, Egypt, January 2000.

[9] S. Nie and Z. Li, "Research and sumulation on valve plate of water hydraulic axial piston pump (motor)," Mechanical science and technology for aerospace engineering, vol. 20, no. 3 , pp. 407-409, 2001.

[10] J. M. Bergada, J. Watton, and S. Kumar, "Pressure, Flow, Force, and Torque between the barrel and port plate in an axial piston pump," Journal of Dynamic Systems, Measurement and Control JANUARY, vol. 130, pp. 1-16, Article ID .011011, 2008.

[11] X. Liu, L. Yu, H. Liu, and K. Jian, "Evaluation of cavitation erosion characteristics in port process of a high-pressure axial piston pump," Mechanical science and technology for aerospace engineering, vol. 27, no. 3, pp. 416-420, 2008.

[12] X. Liu, L. Yu, H. Liu, and K. Jian, "Cavitation erosion mechanism of port plate of hydraulic axial plunger pump," Chinese Journal of Mechanical Engineering, vol. 44, no. 11, pp. 203-208, 2008.

[13] X. Liu, Z. Yang, and W. Wu, "Effect of damping hole on cavitation erosion of port plate of hydraulical axial plunger pump," MACHINE TOOL \& HYDRAULICS, vol. 38, no. 15, pp. 28-31, 2010.

[14] A. Vacca, R. Klop, and M. Ivantysynova, "A numerical approach for the evaluation of the effects of air release and vapour cavitation on effective flow rate of axial piston machines," International Journal of Fluid Power, vol. 11, no. 1, pp. 33-45, 2010.

[15] Z. Jiang and H. Zhou, "Pressure and flow characteristic modeling of water hydraulic axial piston pump based on variable fluid properties," Journal of China Coal Society, vol. 38, no. 1, pp. 171-176, 2013.

[16] C. Liu, X. Wu, and W. Gan, "Numerical simulation of cavitation flow in piston pump based on full cavitation model," China Mechanical Engineering, vol. 26, no. 24, pp. 3341-3347, 2015.

[17] D. Richardson, F. Sadeghi, R. G. Rateick, and S. Rowan, "Dynamic modeling of floating valve plate motion in an axial piston pump," Tribology Transactions, vol. 61, pp. 683-693, 2017.

[18] M. Ivantysynova, C. Huang, and S.-K. Christiansen, "Computer aided valve plate design - an effective way to reduce noise," in Proceedings of the SAE Commercial Vehicle Engineering Congress \& Exhibition, Chicago, IL, USA, 2004.

[19] S. Kuma and J. M. Bergada, "The effect of piston grooves performance in an axial piston pumps via CFD analysis," International Journal of Mechanical Sciences, vol. 66, pp. 168-179, 2012.

[20] J. Zhang, Q. Chao, and B. Xu, "Centrifugal effects on cavitation in the cylinder chamber for high-speed axial piston pumps," Meccanica, vol. 54, 2019.

[21] J. Zhou, A. Vacca, and P. Casoli, "A novel approach for predicting the operation of external gear pumps under 
cavitating conditions," Simulation Modelling Practice and Theory, vol. 45, pp. 35-49, 2014.

[22] G. Changbin, J. Zongxia, and H. Shouzhan, "Theoretical study of flow ripple for an aviation axial-piston pump with damping holes in the valve plate," Chinese Journal of Aeronautics, vol. 27, no. 1, pp. 169-181, 2014.

[23] P. Casoli, M. Pastori, F. Scolari, and M. Rundo, "Active pressure ripple control in axial piston pumps through highfrequency swash plate oscillations-A theoretical analysis," Energies, vol. 12, no. 7, pp. 1-18, Article ID 1377, 2019.

[24] J. Hong and Y. Wang, "Numerical analysis of oil-suction cavitation in cylinder chamber of axial piston pump," Journal of Lanzhou University of Technology, vol. 39, no. 3, pp. 34-37, 2013.

[25] S. Wang, "The analysis of cavitation problems in the axial piston pump," Journal of Fluids Engineering, vol. 132, pp. 1-6, Article ID 074502, 2010.

[26] N. P. Mandal, R. Saha, and D. Sangal, "Effects of flow inertia modelling and valve-plate geometry on swash-plate axial piston pump performance," Proceedings of the Institution of Mechanical Engineers - Part I: Journal of Systems \& Control Engineering, vol. 226, pp. 450-465, 2012.

[27] N. P. Mandal, R. Saha, S. Mookherjee, and D. Sangl, "Pressure compensator design for a swash plate Axial piston pump," Journal of Dynamic Systems, Measurement, and Control, vol. 136, pp. 1-12, Article ID 021001, 2014.

[28] W. Kollek, Z. Kudźma, M. Stosiak, and J. Mackiewicz, "Possibilities of diagnosing cavitation in hydraulic systems," Archives of Civil and Mechanical Engineering, vol. 7, no. 1, pp. 61-73, 2007.

[29] G. Marinaro, E. Frosina, A. Senatore, and K. A. Stelson, “A fast and effective method for the optimization of the valve plate of swash plate Axial piston pumps," ASME Journal of Fluids Engineering, vol. 143, no. 9, pp. 1-13, Article ID 091203, 2021.

[30] Z. Sun, S. Xiao, M. Xu, and W. Wei, "Optimization of the structure of water axial piston pump and cavitation of plunger cavity based on the Kriging model," Journal Vibroengineering, vol. 18, no. 4, pp. 2460-2474, 2016. 